\title{
Religiöse Symbole und Beamtenrecht - Zeit für einen Neustart
}

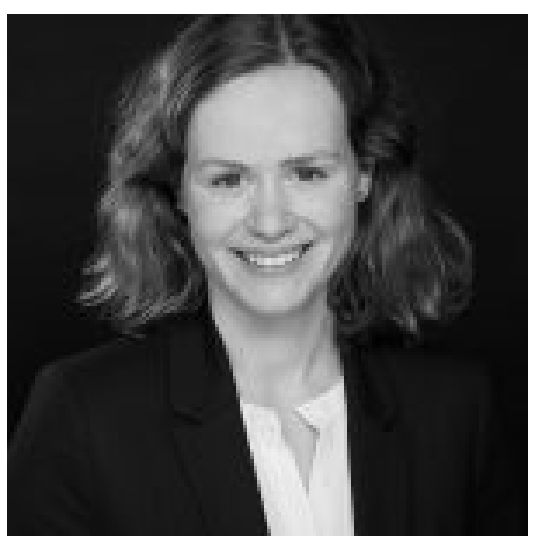

REBEKKA STADLER

Das im Mai 2021 durch Bundestag und Bundesrat verabschiedete Gesetz zur Regelung des Erscheinungsbilds von Beamt:innen soll in Kürze in Kraft treten. Sowohl vor als auch nach seiner Verabschiedung regte sich seitens Islamund Migrationsverbänden Kritik an dem Vorhaben, die bis heute anhält. Das Gesetz verfolgt das begrüßenswerte Ziel, das Vertrauen der Bürger:innen in die Funktionsfähigkeit und Neutralität der staatlichen Verwaltung zu stärken. Im Hinblick auf religiöse Symbole bleibt der Gesetzgeber allerdings auf dem ausgetretenen Weg der Verbote und Beschränkungen, anstatt den verfassungsrechtlich eingeräumten Spielraum für eine pluralitätsfördernde Neugestaltung zu nutzen.

\section{Von der NS-Tätowierung zum potentiellen Verbot religiöser Symbole}

Bundestag und Bundesrat beschlossen im Frühjahr 2021 das Gesetz zur Regelung des Erscheinungsbilds von Beamtinnen und Beamten. Anlass hierfür war der Fall eines mit NS-Zeichen tätowierten Berliner Polizeibeamten. Das BVerwG hatte 2017 die Entfernung des Beamten aus dem Dienst bestätigt, da seine Tätowierungen Ausdruck einer verfassungsfeindlichen Gesinnung gewesen seien - und den Gesetzgeber zugleich angehalten, eine hinreichend bestimmte gesetzliche Grundlage für Vorgaben zum äußeren Erscheinungsbild von Beamt:innen zu schaffen. Diesem Auftrag ist die Regierungskoalition mit entsprechenden Ergänzungen des Beamtenstatusgesetzes (§ 34 Abs. 2 BeamtStG n.F.) und des Bundesbeamtengesetzes (§ 61 Abs. 2 BBG n.F) nachgekommen. Die Neuregelungen erlauben es dem Dienstherrn, das Tragen bestimmter Kleidungsstücke, Symbole und Tätowierungen zu untersagen, „soweit die Funktionsfähigkeit der Verwaltung oder die Pflicht zum achtungs- 
und vertrauenswürdigen Verhalten dies erfordern". Erfreulich ist, dass sich das Parlament des Themas angenommen und die Regelungsproblematik aus dem Dickicht der Verwaltungsvorschriften, Dienstanweisungen und bruchstückhaften Einzelregelungen herausgeholt hat. Aufgrund der Grundrechtssensibilität von Regelungen zum Erscheinungsbild war ein Parlamentsgesetz längst überfällig.

\section{Nicht genutzte Gestaltungsspielräume}

Dennoch steht das Gesetz in der Kritik, und zwar wegen seiner Vorgaben zu religiös konnotierten Erscheinungsmerkmalen. Das mag auf den ersten Blick verwundern, denn eine Einschränkung oder Untersagung solcher Merkmale ist danach nur zulässig, wenn die Symbole objektiv geeignet sind, das Vertrauen in die neutrale Amtsführung der Beamt:innen zu beeinträchtigen. Der Gesetzesbegründung (S. 32) zufolge bezieht sich diese Möglichkeit insbesondere auf Bereiche, in denen der Staat den Bürger:innen gegenüber klassisch-hoheitlich tätig wird, also etwa im Polizeivollzug oder in der Justiz.

Das verabschiedete Gesetz beinhaltet kein unmittelbares Kopftuchverbot für Beamt:innen. Es räumt den Dienstvorgesetzten jedoch die Befugnis ein, dieses in Zukunft auf eine bundesgesetzliche Grundlage zu stützen. Ein Kopftuchverbot in Gerichtssälen und bei der Streifenfahrt mag zwar verfassungsgemäß sein - das klingt in der jüngsten Entscheidung des BVerfG zum Kopftuchverbot für Rechtsreferendarinnen an. In der gleichen Entscheidung gesteht das Gericht dem Gesetzgeber aber einen Einschätzungs- und Gestaltungsspielraum zu, was das Verhältnis von individueller Religionsfreiheit und religiös-weltanschaulicher Neutralität anbelangt. Der Gesetzgeber hätte diesen Spielraum für einen neuen Weg im Umgang mit religiöser Vielfalt nutzen und den Pfad der Verbote verlassen sollen.

\section{Gleichlauf von religiösen Symbolen und unveränderlichen körperlichen Merkmalen}

Als Anwendungsfälle für ein mögliches Verbot von religiös konnotierten Erscheinungsmerkmalen hat der Gesetzgeber laut Gesetzesbegründung (S. 43) z.B. den Polizeivollzug und die Justiz im Blick. Robe und Polizeiuniform betonen in besonderer Weise die amtliche Funktion ihrer Träger:innen, womit religiöse Symbole unvereinbar seien. Und in der Tat: Richter:innen und Polizeibeamt:innen sind in den Augen vieler Bürger:innen geradezu klassische Repräsentant:innen staatlicher Hoheitsgewalt. Darin liegt ein wichtiger Unterschied zu verbeamteten Lehrer:innen, den auch das BVerfG (Rn. 90) hervorhebt. Die Pflicht zum Tragen von Robe und Uniform schließt freilich nicht jegliches individualisiertes Erscheinungsbild aus. Es werden nicht alle persönlichen äußeren Merkmale verdeckt; das unveränderliche Erscheinungsbild bleibt trotz Robe oder Uniform nach außen erkennbar. Geht es um Hautfarbe oder biologisches Geschlecht, ist die Gesellschaft zu Recht bereit, dies bei der Beurteilung der Neutralität der Beamt:innen auszublenden. Doch auch das Tragen eines Kopftuchs ist - wenn man die individuelle religiöse Überzeugung ernst nimmt - für Beamt:innen zwingend und von außen unveränderlich. Das legt es nahe, am Körper getragene religiöse Symbole als Abweichung von einem im 
Übrigen „entindividualisierten“ Erscheinungsbild hinzunehmen - so wie dies bei unveränderlichen körperlichen Merkmalen geschieht.

\section{Zuordnung zum Staat nicht eindeutig}

Aufgrund des hoheitlichen Gewaltmonopols lässt sich der Kontakt zu Polizei und Justiz nicht immer vermeiden. Daher hat der Staat hier in religiös-weltanschaulicher Hinsicht in besonderer Weise Neutralität zu wahren. Bei am Körper getragenen Symbolen ist jedoch - im Unterschied zum Kruzifix in der Amtsstube - die Zuordnung des religiös konnotierten Symbols zum Staat jedenfalls nicht eindeutig. Sowohl in der Gesetzesbegründung als auch in der jüngsten Entscheidung des BVerfG wird angenommen, der Staat müsse sich ein von Beamt:innen am Körper getragenes religiöses Symbol zurechnen lassen. Weder diese Annahme noch die gegenteilige Behauptung, ein am Körper getragenes Symbol sei eindeutig der Privatperson zuzuordnen, ist aber mit Sicherheit belegt - etwa auf der Grundlage entsprechender Studien. Solange die Frage nach der Zuordnung zum Staat nicht substantiiert beantwortet ist, steht dem Gesetzgeber hinsichtlich der Eignung eines Kopftuchverbots zur Wahrung staatlicher Neutralität eine Einschätzungsprärogative zu. In Ansehung dieses Einschätzungsspielraums mag ein Kopftuchverbot zulässig sein. Dennoch: in einer aufgeklärten und diversen Gesellschaft sollte der Staat seinen Bürger:innen im Zweifel zutrauen, ein religiöses Symbol seinem Träger als Privatperson zuzuordnen. Mit anderen Worten: Der Staat wahrt die gebotene religiös-weltanschauliche Neutralität auch dann, wenn er religiös konnotierte Symbole nicht verbietet und hierdurch in die Religionsfreiheit seiner Beamt:innen eingreift.

\section{Vertrauen stärken durch gelassene Akzeptanz}

Ziel der Bundesregierung ist es, mit dem neuen Gesetz das Vertrauen in die Neutralität der Verwaltung zu stärken. Dafür wäre ein anderer Weg als der eingeschlagene aber zielführender. Denn das Vertrauen in eine sachgerechte Amtsführung hängt auch davon ab, dass Bürger:innen sich repräsentiert fühlen. Die zunehmende Diversität in der Gesellschaft sollte sich daher auch unter den Staatsbediensteten wiederfinden - nicht nur an Schulen, sondern auch in Polizei und Justiz. Nicht ohne Grund hat Gärditz das Berufsbeamtentum unlängst als Institution beschrieben, in der „selbstbewusste Bürgerinnen und Bürger gerade in ihrer Unterschiedlichkeit gemeinsam Verantwortung für ihren Staat übernehmen“. Durch ein Kopftuchverbot für Beamt:innen würde ein Teil der Bevölkerung von bestimmten Ämtern faktisch ausgeschlossen. Das schadet der Diversität und schwächt die Repräsentations- und Identifizierungsmöglichkeiten einzelner Bevölkerungsgruppen.

In der verfassungsrechtlichen Diskussion um Kopftuchverbote für Beamt:innen wird oft aus dem Blick verloren, dass jedes Amt durch einen Menschen ausgeübt wird, der bestimmte Einstellungen, eine persönliche Biographie, politische Auffassungen und religiöse Überzeugungen in sich trägt. Dieser persönlichen „Konfiguration“ werden gut ausgebildete Beamt:innen keinen Einfluss auf ihre dienstlichen Entscheidungen einräumen. Darauf dürfen die Bürger:innen im Grundsatz vertrauen. Nichts anderes sollte für den Fall gelten, dass eine Polizistin 
ein Kopftuch trägt oder ein Richter mit Kippa auftritt. Wo das Vertrauen enttäuscht wird und Staatsdiener:innen sich von ihren Überzeugungen leiten lassen, bietet das Disziplinarrecht geeignete Reaktionsmöglichkeiten.

\section{Zeit für einen religionspolitischen Neustart im Beamtenrecht}

Mit der vom Gesetzgeber beabsichtigten Stärkung des gesellschaftlichen Vertrauens in die Neutralität der Verwaltung geht in der gewählten Form ein doppeltes Misstrauensvotum einher: Zum einen traut der Gesetzgeber es den Bürger:innen nicht zu, zwischen dem äußeren Erscheinungsbild der Amtswalter:innen und der hiervon unbeeinflussten Amtsausübung zu unterscheiden. Zum anderen vermittelt das Gesetz den Eindruck, gewissen Ressentiments gegenüber muslimischen Frauen eine Legitimation zu verleihen. Anstatt einen zeitgemäßen und respektvollen Umgang mit religiöser Pluralität zu fördern und dieser Diversität auch in Polizei und Justiz mit der nötigen Gelassenheit zu begegnen, werden durch das Gesetz bestimmte Bevölkerungsgruppen vom Zugang zu Ämtern ausgeschlossen.

Im Spannungsverhältnis von religiös-weltanschaulicher Neutralität des Staates und der individuellen Religionsfreiheit von Beamt:innen hat der Gesetzgeber die Chance für einen Neustart verpasst. Der verfassungsrechtliche Spielraum sollte durch den Gesetzgeber für ein deutliches Signal an die Bürger:innen genutzt werden: Von Beamt:innen getragene religiöse Symbole schließen eine sachgerechte und neutrale Amtsführung nicht aus.

Zitiervorschlag: Rebekka Stadler, Religiöse Symbole und Beamtenrecht - Zeit für einen Neustart, JuWissBlog Nr. 72/2021 v. 1.7.2021, https://www.juwiss.de/72-2021/

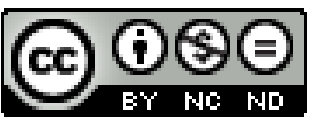

Dieses Werk ist lizenziert unter einer Creative Commons Namensnennung - Nicht kommerziell - Keine Bearbeitungen 4.0 International Lizenz. 